\title{
Das betriebliche Eingliederungsmanagement - Ein doppelter Suchprozess
}

\section{ETABLIERUNG DES BETRIEBLICHEN EINGLIEDERUNGSMANAGEMENTS}

Seit 2001 wurden die Instrumente des Rehabilitationsrechts modernisiert; präventive und betriebliche Maßnahmen wurden ausgebaut bzw. neu etabliert. Ein wichtiges Instrument ist das Betriebliche Eingliederungsmanagement (BEM), das 2001 eingeführt und 2004 nachhaltig umgestaltet wurde. Danach ist der Arbeitgeber verpflichtet, allen Beschäftigten, die in den letzten zwölf Monaten mindestens sechs Wochen arbeitsunfähig waren, ein strukturiertes betriebliches Eingliederungsmanagement anzubieten. Bereits in den parlamentarischen Beratungen ist die Bedeutung der Gesundheitsprävention betont worden:

„Durch die gemeinsame Anstrengung aller Beteiligten soll ein betriebliches Eingliederungsmanagement geschaffen werden, das durch geeignete Gesundheitsprävention das Arbeitsverhältnis möglichst dauerhaft sichert. Viele Abgänge in die Arbeitslosigkeit erfolgen immer noch aus Krankheitsgründen. ... Die Regelung verschafft der Gesundheitsprävention am Arbeitsplatz dadurch einen stärkeren Stellenwert, dass die Akteure unter Mitwirkung des Betroffenen zur Klärung der betreffenden Maßnahmen verpflicht werden“.

Diese Etablierung war zumindest medial sehr erfolgreich. Mit insgesamt mehr als 100 Urteilen vor allem der Arbeitsgerichtsbarkeit, darunter inzwischen wenigstens zehn Urteile des Bundesarbeitsgerichts, hat dieses Verfahren eine deutlich höhere Aufmerksamkeit gefunden als die knapp zehn Jahre vorher eingeführte Gefährdungsbeurteilung nach $₫ 5 \mathrm{ArbSchG}$.

Mit der griffigen Formulierung „BEM“ ist dieses Verfahren inzwischen - wiederum anders als die Gefährdungsbeurteilung - fast allen Richterinnen und Richtern der Arbeitsgerichtsbarkeit sowie einem
Großteil der Betriebsräte und Personalleitungen bekannt. Eine wichtige Untersuchung, an der vor allem in diesem Feld engagierte Betriebe teilgenommen haben, dokumentiert Chancen und Verfahren des BEM. ${ }^{2}$ Zugleich zeigen erste Erfahrungen sowie die große Mehrzahl der arbeitsgerichtlichen Verfahren, dass in der betrieblichen Praxis noch viele Unklarheiten bestehen. $^{3}$

Mit der Einführung des BEM ist ein doppelter Suchprozess installiert worden, der noch lange nicht abgeschlossen ist. Es sind keine neuen Sachnormen des Arbeitsschutz- und Gesundheitsrechts geschaffen worden; vielmehr ist eine Verfahrenspflicht des Arbeitgebers normiert worden, die ihm gebietet, zusammen mit den betrieblichen Interessenvertretungen ein geeignetes Verfahren zu regeln, das den Beschäftigten zu erläutern und anzubieten ist. ${ }^{4}$ Adressat sind alle Beschäftigten, die in den letzten zwölf Monaten wenigstens sechs Wochen arbeitsunfähig waren, ohne dass es auf die Ursachen der Arbeitsunfähigkeit oder den Status als schwerbehinderter Mensch ankommt. Damit ist die Eingangsschwelle für das Verfahren einfach formuliert. Während der Arbeitgeber verpflichtet ist, dieses Verfahren einzuführen, sind die Beschäftigten frei, ob sie der Einleitung des Verfahrens zustimmen. Selbst wenn sie eine solche Zustimmung erteilt haben, kann diese von ihnen jederzeit widerrufen werden, da sie die „Herren des Verfahrens“ sind. ${ }^{5}$ Damit ist BEM klar abzugrenzen von den bisherigen Krankenrückkehrgesprächen. ${ }^{6}$

\section{BEM ALS ORGANISIERTER UND KOOPERATIVER SUCHPROZESS}

Mit dem Betrieblichen Eingliederungsmanagement (BEM) sollen gesundheitspräventive Maßnahmen realisiert werden. Um die geeigneten Maßnahmen finden zu können, steht am Anfang dieses Weges die Suche nach den betrieblichen Defiziten und Problemlagen. Da die Probleme und vor allem die Maßnahmen selten unmittelbar auf der Hand liegen, bedarf es einer organisierten Suche. Mein Vorschlag, das BEM als „organisierten Suchprozess“ zu qualifizieren, ist inzwischen vom Bundesarbeitsgericht (BAG) aufgegriffen worden.? Damit die verschiedenen Akteure erfolgreich zusammenwirken, ist des Weiteren ein „kooperativer Prozess“ erforderlich. ${ }^{8}$

\section{NOTWENDIGE AKTEURE}

Die Verfahrenspflicht richtet sich an den Arbeitgeber, erster Adressat ist der betrof-

\footnotetext{
Bundestagsdrucksache 15/1783, S. 16

2 Niehaus, M. (2008): Betriebliches Eingliederungsmanagement, Bonn.

3 Kohte, W. (2009): Krankheitsbedingte Kündigung und BEM, in: Arbeitsrecht im Betrieb (AiB) 6, S 385-388.

4 Faber, U. (2008): Was Betriebe für Langzeitkranke tun müssen: Auswirkungen des BEM auf das Arbeits- und Kündigungsschutzrecht, in: Soziale Sicherheit 4, S. 130-133.

5 Gagel, A. (2004): Betriebliches Eingliederungsmanagement, in: Neue Zeitschrift für Arbeitsrecht (NZA) 24, S. 1359-1362.

6 Oppolzer, A. (2010): Gesundheitsmanagement im Betrieb: Integration und Koordination menschengerechter Gestaltung der Arbeit, Hamburg.

7 Kohte, W. (2008): Betriebliches Eingliederungsmanagement und Bestandsschutz, in: Der Betrieb 11, S. 582-587.; ausdrücklich BAG 10.12.2009/2 AZR 400/08.
}

8 KSW/Kohte SGB IX, §84, Rn. 31. 
fene Beschäftigte, sodass diese beiden in jedem Fall die unverzichtbaren Akteure darstellen. Weiter sind als unverzichtbar qualifiziert - soweit im Betrieb vorhanden ${ }^{9}$ - die betriebliche Interessenvertretung (Betriebs- und Personalräte) sowie bei schwerbehinderten Menschen die Schwerbehindertenvertretung. Das Gesetz verschafft diesen ein originäres Teilhaberecht, das sie arbeitsgerichtlich durchsetzen können. Sie können die Ausgestaltung des jeweiligen Verfahrens und die möglichen Maßnahmen nachhaltig beeinflussen.

\section{OPTIONALE AKTEURE}

Alle weiteren Akteure haben kein obligatorisches, sondern nur ein optionales Teilhaberecht. Ausdrücklich genannt werden in $\$ 84$ Abs. 2 Satz 2 Sozialgesetzbuch (SGB) IX die „Werks- und Betriebsärzte“. Diese gehören - was für den Charakter des BEM von Bedeutung ist - nicht zu den unverzichtbaren Akteuren, sondern werden nur bei Erforderlichkeit hinzugezogen. Dies entspricht der Stellung des Betriebsarztes als Berater der Betriebsparteien (dazu $\$ \$ 3,9$ Gesetz über Betriebsärzte, Sicherheitsingenieure und andere Fachkräfte für Arbeitssicherheit (ASiG)), der keine eigenen Entscheidungen zu treffen hat. Diese Abstufung zeigt aber vor allem, dass die Ergebnisse des BEM vorrangig nicht auf medizinische, sondern auf betriebliche Maßnahmen zielen. Aus diesem Grund gehören zu den optionalen Akteuren auch die Sicherheitsfachkräfte nach $\S 5 \mathrm{ff}$. ASiG und im Einzelfall auch Sicherheitsbeauftragte nach $\$ 22$ SGB VII.

Während die internen Akteure zumindest in gut organisierten Betrieben regelmäßig im Arbeitsschutzausschuss nach $\S 11$ ASiG zusammenkommen, ist die Pflicht zur Einbeziehung externer Akteure eine verfahrensrechtliche Innovation. In allen Fällen, in denen sozialrechtliche Hilfen in Betracht kommen und die Beteiligen selbst keine abschließende Lösung finden können, sind externe Experten, vor allem die Servicestellen der Rehabilitationsträger und die Integrationsämter, in das Verfahren einzubeziehen. Solche Hilfen kommen oft in Betracht, denn sowohl Hilfsmittel und technische Arbeitshilfen als auch Maßnahmen der medizinischen Rehabilitation oder der Arbeitsassistenz können in einer beachtlichen Zahl von Fällen eingesetzt werden. Ein aktueller Bericht des Büros für Technikfolgenabschätzung beim Deutschen Bundestag hat zahlreiche Beispiele dokumentiert; ${ }^{10}$ sie zeigen zugleich, dass in Deutschland produzierte Hilfsmittel zwar einen hohen Exportanteil haben, im Inland dagegen deutlich seltener eingesetzt werden. Diese Lücke zwischen technischem Know-how und organisatorischen Defiziten kann ein professionelles BEM-Verfahren verkleinern. Die Rehabilitationsträger haben sich in einer Gemeinsamen Empfehlung vom 16.12.2003 verpflichtet, BEM-Verfahren zu unterstützen und die Beteiligten zu beraten. In der jüngsten Entscheidung des BAG zu den verfahrensrechtlichen Mindeststandards gehört die Einbeziehung aller zuständigen Stellen, also auch der externen Experten, zu den elementaren Standards, an denen ein BEM zu messen ist.

\section{ZIEL DES SUCHPROZESSES}

Der Gesetzestext wird teilweise in der Weise missverstanden, dass durch ein Betriebliches Eingliederungsmanagement (BEM) die Betroffenen therapiert und geheilt werden können. Dies ist mit dem Begriff der Überwindung der Arbeitsunfähigkeit jedoch nicht gemeint, denn das Verfahren ist kein medizinisches, sondern ein betriebliches Eingliederungsmanagement. Dies zeigt sich bereits in der Zusammensetzung der Akteure: Ärzte sind optionale Akteure, Arbeitgeber und Betriebsrat sind dagegen wegen ihrer Kenntnis des Betriebes und der Fähigkeit, betriebliche Gestaltungsmöglichkeiten zu ermitteln und zu realisieren, für das Verfahren unverzichtbar. ${ }^{11}$ Zutreffend nennt daher das BAG als vorrangige Aufgabe die Anpassung des Arbeitsplatzes und, soweit diese nicht möglich ist, die Suche nach einem anderen leidensgerechten Arbeitsplatz. Dagegen sind die bisherigen Krankenrückkehrgespräche vom BAG zutreffend als eine Kontrollmaßnahme qualifiziert und der Mitbestimmung nach $\$ 87$ Abs. 1 Ziff. 1 Betriebsverfassungsgesetz (BetrVG) unterworfen worden. Für das BEM gelten dagegen die Regeln für integrative Verfahren. ${ }^{12}$

\section{SCHRITTWEISER SUCHPROZESS}

Geboten ist es daher, bereits vor oder in einem Erstgespräch die Gefährdungsbeurteilung des jeweiligen Arbeitsplatzes heranzuziehen, um mögliche Problemlagen, aber auch Anpassungsmöglichkeiten finden zu können. In denjenigen BEM-
Gerichtsverfahren, in denen ergonomische Gutachten eingeholt wurden, ergaben sich nicht selten beachtliche Gestaltungsmöglichkeiten, die von den betrieblichen Akteuren bis zu diesem Zeitpunkt nicht erkannt worden waren. ${ }^{13}$ Weniger günstig waren bisher die Erfahrungen mit medizinischen Gutachten, die in der Regel nur die Leistungsdefizite der jeweiligen Beschäftigten formulierten, während ihre Ressourcen und die sich daraus ergebenden Anpassungsaufgaben und -chancen am Arbeitsplatz kaum in den Blick gerieten. Im ersten Grundsatzurteil zur Bedeutung des BEM für das Kündigungsrecht hat der 2. Senat des BAG eine solche defizitorientierte Begutachtung nachhaltig kritisiert. ${ }^{14}$

\section{WECHSELWIRKUNGEN ZUM ARBEITSSCHUTZRECHT}

In der Mehrzahl der BEM-Verfahren, die an das BAG gelangten, war zu konstatieren, dass Wirbelsäulenprobleme vorlagen und dass die Arbeitsplätze den arbeitsschutzrechtlichen Anforderungen der Lasthandhabungsverordnung widersprachen. Nach dieser Verordnung ist die Handhabung von Lasten grundsätzlich zu vermeiden bzw. zu verringern. In jedem Fall ist der Einsatz von Hilfsmitteln zu prüfen und soweit möglich zu realisieren. ${ }^{15}$ BEM ist ein mögliches und aus meiner Sicht effizientes Verfahren zur Aufdeckung arbeitsschutzrechtlicher Defizite im Betrieb.

Ein anschauliches Beispiel liefert ein Fall, der am Landesarbeitsgericht (LAG) Köln verhandelt wurde. Ein Gepäckabfertiger am Flughafen war gekündigt worden, als seine Zeiten der Arbeitsunfähigkeit nach zehn Jahren Betriebszugehörigkeit deutlich

9 Zum BEM im Kleinbetrieb: LPK-SGB IX-Düwel § 84 Rn. 48.

10 Gerlinger, K./Revermann, C. (2009): Chancen und Perspektiven behinderungskompensierender Technologien am Arbeitsplatz, Berlin.

11 Schlewing, A. (2005): Das betriebliche Eingliederungsmanagement nach § 84 Abs. 2 SGB IX - Keine Wirksamkeitsvoraussetzung für die Kündigung wegen Krankheit, in: Zeitschrift für Arbeitswissenschaft (ZfA) 4, S. 485-503.

12 Feldes, W. (2009): Vom Fehlzeiten- zum Eingliederungsmanagement, in: Arbeitsrecht im Betrieb (AiB) 4, S. 222-227.

13 Zum Beispiel: BAG NZA 2001, 1020; 2006, 442.

14 BAG NZA (2008), S. 173.

15 Zipprich, J. (2006): Prävention arbeitsbedingter Erkrankungen durch manuelles Handhaben von Lasten: Rechtliche Anforderungen und Perspektiven nach Umsetzung der Richtlinie 90/269/EWG, Baden-Baden. 
zugenommen hatten. Im Prozess stellte sich heraus, dass er beachtliche Lasten zum Teil in ungünstiger Haltung zu bewegen hatte. Im Betrieb bestand eine Regelung, wonach Arbeitnehmer, die einen Wirbelsäulenschaden nachweisen konnten, Tragehilfen erhielten. Trotzdem war dem Kläger keine Hilfe gewährt worden, sodass das LAG Köln die Kündigung als unwirksam qualifiziert hatte, weil der Einsatz von Hebehilfen ein mögliches Mittel darstelle, um die Weiterbeschäftigung ohne überdurchschnittliche Krankheitszeiten zu sichern.

In diesem Fall war kein BEM durchgeführt worden, was das Gericht auch gerügt hatte. Hätte man dieses Verfahren durchgeführt, dann wäre spätestens mithilfe externen Sachverstands ermittelt worden, dass die bisherige betriebliche Regelung das Arbeitsschutzrecht auf den Kopf gestellt hat. Im präventiven Arbeitsschutz werden Hebehilfen eingesetzt, um Wirbelsäulenschäden zu verhindern. Auf keinen Fall ist es sachgerecht, mit deren Einsatz so lange zu warten, bis ein solcher Schaden nachgewiesen werden kann. ${ }^{16}$

In dem Kölner Gerichtsverfahren war von keinem der Beteiligten thematisiert worden, ob überhaupt eine Gefährdungsbeurteilung für diesen Arbeitsplatz erstellt worden war. Den bekannten Umsetzungsdefiziten ${ }^{17}$ würde es entsprechen, dass eine solche Beurteilung bisher fehlte; das BEMVerfahren ist eine Chance, diese „weißen Flecken“ des präventiven Arbeitsschutzes im Betrieb aufzudecken und schrittweise zu verringern.

Denkbar ist es aber auch, dass eine schematische Beurteilung mit einer Checkliste durchgeführt worden ist, die den antipräventiven Einsatz der Tragehilfen verdeckt hatte. Nach $\$ 3$ ArbSchG ist jeder Arbeitgeber verpflichtet, nicht nur Gefährdungsbeurteilungen zu erstellen, sondern diese auch regelmäßig zu evaluieren. Nach unseren bisherigen Erfahrungen erfolgt eine solche Evaluation selten.

Nach meiner Ansicht sind BEM-Verfahren, die fair durchgeführt werden, ein besonders gut geeignetes Mittel, um Gefährdungsbeurteilungen zu erstellen bzw. $\mathrm{zu}$ evaluieren. In der bisherigen betrieblichen Praxis ist in Deutschland das Verständnis für ganzheitliche Gefährdungsbeurteilungen relativ gering. In der Welt des vorschriftenorientierten Arbeitsschutzes ist Ausgangspunkt nicht der konkrete Arbeitsplatz, sondern eine abstrakte und generelle Norm, die es umzusetzen gilt.
Dieser vorschriftenorientierte Arbeitsschutz befindet sich - zu Recht - seit einigen Jahren in der Defensive. Er ist nur selten in der Lage, aktiv auf Änderungen der Arbeitsplätze einzuwirken, sondern bleibt reaktiv und erschwert jegliche Form von Partizipation und Innovation durch die Beschäftigten. Die Methode der Gefährdungsbeurteilung, die vom konkreten Arbeitsplatz ausgeht, ist dem Weltbild des traditionellen Arbeitsschutzes fremd; viele der bei uns üblichen Formulare und Checklisten für Gefährdungsbeurteilungen motivieren und befähigen Beschäftigte wenig zu einer solchen Partizipation.

BEM-Verfahren beginnen immer konkret, weil es um die Arbeitsunfähigkeit konkreter Personen an konkreten Arbeitsplätzen geht. Wenn dieses Verfahren mit präventiven Methoden durchgeführt wird, kann es alle Beteiligten zur gemeinsamen Arbeit an realistischen und betriebsnahen Gefährdungsbeurteilungen und vor allem zur Entwicklung und Realisierung konkreter Maßnahmen zur Gestaltung der Arbeitsplätze mobilisieren.

\section{BEM ALS GRATWANDERUNG}

Eine solche präventive Orientierung ist zwar notwendig und chancenreich, bisher aber noch wenig praktiziert. Solange Beschäftige damit rechnen müssen, dass die Informationen aus dem BEM-Verfahren im Rahmen einer Krankheitskündigung genutzt werden können, werden sie zu Recht misstrauisch sein und das Verfahren nur als eine kaschierte Form der Krankenrückkehrgespräche ansehen. Der Leitbildwechsel ist schwierig; aus der Praxis wird berichtet, dass in nicht wenigen Betrieben nebeneinander Betriebsvereinbarungen zu Krankenrückkehrgesprächen und Absprachen zur Durchführung eines BEM bestehen. ${ }^{18}$ Auf diese Weise wird eine effektive Gesundheitsprävention nicht erreicht werden können. Nach der Rechtsprechung des BAG ist die rechtliche Lösung einfach: Krankenrückkehrgespräche unterliegen der Mitbestimmung des Betriebsrats, der eine etwaige Betriebsvereinbarung mit einer Frist von drei Monaten ( $\$ 77$ BetrVG) oder zum nächsten vereinbarten Termin kündigen kann. Spätestens in der Einigungsstelle wird das bisherige kontrollierende Krankenrückkehrgespräch schwerlich mehrheitsfähig sein, weil das heutige Arbeitsschutz- und Rehabilitationsrecht Prävention als vorrangigen Weg verlangt.
Wenn der bisherige Kontrollweg verlassen werden soll, dann ist es unverzichtbar, effektive Regelungen zum Datenschutz $\mathrm{zu}$ vereinbaren. Dazu liegen mehrere gut handhabbare Vorschläge vor. ${ }^{19}$ Sie gehen vom Prinzip der Datensparsamkeit mit einem abgestuften Verfahren aus, das zunächst mit einem Erstgespräch in einem kleinen Kreis beginnt und personenunabhängige Daten, wie z. B. die betriebliche Gefährdungsbeurteilung, heranzieht oder erstellt. Erst auf dieser Basis kann es im Einzelfall sinnvoll sein, individuelle medizinische Daten zu ermitteln, wenn der generelle Arbeitsschutz allein nicht ausreicht. Die in der arbeitswissenschaftlichen Diskussion formulierte Priorität zwischen dem universellen und barrierefreien Design einerseits und den individuellen assistiven Technologien andererseits ${ }^{20}$ gilt auch in diesem Zusammenhang.

Als ein erfolgreiches integratives Verfahren hat sich die stufenweise Wiedereingliederung nach $\$ 28$ SGB IX erwiesen. Nach einer nachhaltigen Arbeitsunfähigkeit ist es nicht selten der Fall, dass die Beschäftigten nicht ohne Weiteres ihre bisherige Arbeit wieder aufnehmen können. Das aussperrende Verhalten, die Beschäftigten möglichst lange von ihrem bisherigen Arbeitsplatz fernzuhalten, hat sich nur in wenigen Fällen als gesundheitsfördernd erwiesen. In der Mehrzahl der Fälle ist die stufenweise Eingliederung der am besten geeignete Weg, um die Integration in den Arbeitsmarkt zu sichern. Nach der neuen Rechtsprechung des BAG ${ }^{21}$ kann der Arbeitgeber zu einer solchen Mitwirkung und Zustimmung verpflichtet sein, wenn ein geeigneter Eingliederungsplan vorliegt. Ein solcher Plan ist nur schwer von Hausärzten aufstellbar, weil diesen die betrieblichen und sozialmedizinischen Kenntnisse oft fehlen. ${ }^{22}$ Es bedarf daher des

16 Kohte, W. (2009): Krankheitsbedingte..., a.a.O 17 Vgl. Beitrag von Rolf Satzer in diesem Heft. 18 Feldes, W. (2009), a.a.O.

19 Gundermann, L./Oberberg, M. (2007): Datenschutzkonforme Gestaltung des betrieblichen Eingliederungsmanagements und Beteiligung des Betriebsrates, in: Arbeit und Recht (AuR) 1, S. $19-26$.

20 Gerlinger, K./Revermann, C. (2009), a.a.O

21 BAG NZA (2007), S. 91; zu den Einzelheiten FKS-SGB IX/Nebe § 28 Rn. 16.

22 Denkbar und sinnvoll wäre es, wenn alle Beschäftigten eine Kopie ihrer Gefährdungsbeurteilung haben und diese ihren Ärzten vorlegen können. Trotz der Dokumentationspflicht des § 6 ArbSch C erscheint dies in Deutschland allerdings noch futuristisch zu sein. 
Zusammenwirkens von Arbeitnehmer, Betriebsrat und Arbeitgeber, möglicherweise unterstützt durch Hausarzt, Betriebsarzt und den Arzt in der jeweiligen Rehabilitationseinrichtung. Dieses Zusammenwirken ist nicht nur einmalig erforderlich, denn die stufenweise Wiedereingliederung ist ein Prozess, sodass der Eingliederungsplan jeweils nachjustiert werden muss. ${ }^{23}$ In der Praxis der gut organisierten Betriebe ist die stufenweise Wiedereingliederung die am häufigsten praktizierte Maßnahme, die im BEM-Verfahren vereinbart wird. Sie dokumentiert die Chancen eines solchen kooperativen Suchprozesses und die integrativen Möglichkeiten der Betriebsärzte in der betrieblichen Sozialverfassung. ${ }^{24}$

\section{BETRIEBSVERFASSUNG ALS MEDIUM DER VERFAHRENSORDNUNG}

Ein solcher Prozess bedarf einer allgemeinen Verfahrensordnung. ${ }^{25}$ Da das Betriebliche Eingliederungsmanagement (BEM) auch eine Maßnahme des verpflichtenden Gesundheitsschutzes darstellt, weil der Arbeitgeber gehalten ist, dieses Verfahren durchzuführen, ist hier das Mitbestimmungsrecht nach $\$ 87$ Abs. 1 Ziff. 7 BetrVG zu bejahen. ${ }^{26}$ In der arbeitsrechtlichen Literatur sind die verschiedenen Elemente bereits hinreichend klar beschrieben worden: Die Regelung der Beteiligung interner und externer Personen, die Bildung eines Integrationsteams, die Sicherung des Arbeitnehmerdatenschutzes, die Abstufung des Verfahrens in Erstgespräch und weitergehende Formen der Klärung mit weiteren Beteiligten; die Nutzung und Verbesserung der jeweiligen Gefährdungsbeurteilung; die obligatorische Prüfung des Einsatzes von Hilfsmitteln, Arbeitsassistenz und stufenweiser Wiedereingliederung. ${ }^{27} \mathrm{Im}$ ersten BEM-Beschlussverfahren am BAG fehlten konkrete Regelungsvorschläge, sodass der Antrag des Betriebsrats bereits aus prozessrechtlichen Gründen scheitern musste. ${ }^{28}$ Der 1 . Senat des BAG beschränkte sich auf Hinweise, welche Regelungskomplexe möglich sind. Es ist Sache der Beteiligten, die für ihren Betrieb passenden konkreten Regelungen zu ermitteln. Dieses Umdenken ist schwierig, sodass sich auch engagierte Betriebsräte noch in einem Suchprozess befinden, wie sie für ihren Betrieb das BEM ausgestalten sollen. Es findet insoweit ein Suchprozess nach den geeigneten Formen des BEM-Suchprozes- ses statt. Das jüngste Urteil des 2. Senats des BAG, das konkrete verfahrensrechtliche Mindeststandards verlangt, könnte auch für Betriebsräte ein Anlass sein, die Suche nach dem für ihre betriebliche Situation geeigneten Verfahren aufzunehmen oder zu intensivieren und auf diesem Weg zugleich den präventiven Arbeitsschutz in ihrem Betrieb zu fördern. Damit ist BEM nicht nur ein Suchprozess für den einzelnen Beschäftigten, sondern auch ein Suchprozess für Betriebs- und Personalräte zur Realisierung präventiven Arbeitsschutzes in ihrem Betrieb.

23 Nebe, K. (2008): (Re-)Integration von Arbeitnehmern: Stufenweise Wiedereingliederung und Betriebliches Eingliederungsmanagement - ein neues Kooperationsverhältnis, in: Der Betrieb 33, S. $1801-1805$.

24 Kohte, W. (2010): Betriebsärzte zwischen Reduktion, Prävention und Integration, in: Gerlinger et al.: Politik für Gesundheit, Bern, S. 280-290.

25 Schlewing, A. (2005): a.a.O.

26 Kohte, W. (2009): Krankheitsbedingte..., a. a. O.; Schils, M. (2009): Das Betriebliche Eingliederungsmanagement im Sinne des §84 Abs. 2 SGB IX, Frankfurt.

27 Zuletzt Oppolzer, A. (2010): a.a.O.

28 BAG 18.08.2009-1 ABR 45/08.

\section{Eine vorausschauende, ganzheitliche Gefährdungsbeurteilung}

\begin{abstract}
Zahlreiche betriebliche Erfahrungen belegen, dass sich die ganzheitliche, unter Einbeziehung psychischer Belastungen durchgeführte Gefährdungsbeurteilung nach $\$ 5$ Arbeitsschutzgesetz (ArbSchG) als zentrales Präventionsinstrument im modernen Arbeits- und Gesundheitsschutz bewährt hat. ${ }^{1}$ Die bislang praktizierten Ansätze der Gefährdungsbeurteilung setzen an vorhandenen Arbeitsbedingungen an, ermitteln und beurteilen Gefährdungen und Belastungen und umfassen ebenso die Ableitung und Umsetzung von Maßnahmen menschengerechter Arbeitsgestaltung. Wie kann nun der Präventionsansatz des Gesetzes für eine Gestaltung der Arbeitsbedingungen schon im Planungsstadium, z. B. vor oder in Umstrukturierungspro-
\end{abstract}

zessen, im Sinn einer vorausschauenden Gefährdungsbeurteilung stärker genutzt werden, die nicht nur den Ist-Zustand, sondern bereits den vom Arbeitgeber geplanten Soll-Zustand bewertet und rechtzeitig beeinflusst?

\section{UMSETZUNGSDEFIZITE UND POSITIVE PRAXISERFAHRUNGEN}

Auch wenn positive Erfahrungen mit der Durchführung von Gefährdungsbeurteilungen unter Einbeziehung psychischer Belastungen vorliegen, ist zu betonen, dass dieses Präventionsinstrument in Unternehmen und Betrieben nur unzureichend umgesetzt wird. Tatsächlich gibt es in der Praxis erhebliche Defizite im Hinblick auf die Realisierung der ganzheitlichen Gefährdungsbeurteilung. 2003 gingen Expertenschätzungen davon aus, dass in lediglich

\footnotetext{
Langhoff, T./Satzer, R./Ertel, M. (2010): Erfahrungen zur Umsetzung der Gefährdungsbeurteilung bei psychischen Belastungen, Forschungsprojekt im Auftrag der Bundesanstalt für Arbeitsschutz und Arbeitsmedizin (BAuA) in: Arbeit 2010, Veröffentlichung in Vorbereitung; online: www. gefaehrdungsbeurteilung-forschung.de.
}

Rolf Satzer, Diplom Psychologe, arbeitet freiberuflich an umsetzungsorientierten Forschungsprojekten. Arbeitsschwerpunkte: Gesundheit, Qualifizierung, Arbeitsbedingungen, Technologie. e-mail: rolf.satzer@t-online.de 